\title{
Flow design in photonic data transport network
}

\author{
Mateusz Dzida \\ Optimax \\ ul. Wolbromska 19/A, 03-680 Warszawa, Poland \\ Email: mdzida[at]onet.eu
}

\author{
Andrzej Bąk \\ Optimax \\ ul. Wolbromska 19/A, 03-680 Warszawa, Poland \\ Email: abak[at]poczta.pl \\ Warsaw University of Technology \\ ul. Nowowiejska 15/19, 00-665 Warszawa, Poland \\ Email: bak[at]tele.pw.edu.pl
}

\begin{abstract}
Development of sophisticated photonic transmission systems enabled evolution of photonic data transport networks towards cost-efficient and energy-efficient platforms capable to carry enormous traffic. Given access to technologically advanced equipment, network operator faces a series of decision problems related to how to efficiently use this technology. In this paper, we propose a functional model of modern photonic network with wavelength division multiplexing (WDM). Proposed functional model is a basis for formulating corresponding flow design problem in terms of mathematical programming.
\end{abstract}

\section{INTRODUCTION}

$\mathbf{R}$ ECENT advances in the photonic networking enabled rapid growth of the transmission rates in the modern photonic data transport networks. Thus, photonic data transport networks became considerable alternative for traditional electric-based transmission systems, and are more and more widely deployed in the Autonomous Systems composing the Internet.

An important area of research in the domain of photonic data transport networks is associated with development of functional models of photonic networks and mathematical models of the decision problems associated with designing such networks.

Modeling telecommunication network is not a trivial task. On one hand, development of functional models requires detailed knowledge of transmission technology and networking protocols. Thus, such functional models must be sufficiently detailed to accurately represent costs of equipment. On the other hand, it is necessary to consider a number of specific aspects that may further turn into design constraints. Most important of these include:

- architecture of switching equipment and transmission,

- mechanisms for traffic grooming and consolidation,

- network reconfiguration in case of failure,

- physical effects associated with signal propagation.

In the balance of this paper we propose mathematical (optimization) model of the flow design problem related to photonic data transport network. Developed model is expressed in terms of integer programming. It refers to generic input data,

This work presents results of realization of first phase of ongoing project "Optymalizacja przepływów w wielowarstwowych sieciach dostepu do Internetu w technologii DWDM/IP/MPLS" (ODIN). Project is co-founded by The National Centre for Research and Development, Poland. including: network topology, infrastructure, and considered products. In particular, on one hand, input data must determine full cost characteristics of the considered equipment, usually in form of cost of particular expansion cards. On the other hand, input data are supposed to include locations of client devices and their demand for data transport services. It is therefore assumed that knowledge possessed by a network operator about demand structure is certain. In practice, knowing exact demand for transport services can be difficult, and sometimes even impossible. Still, we assume that through appropriate statistical methodology, it is possible to determine demand value with reasonable degree of confidence.

Paper is organized as follows. In Section II we present general principles of photonic data transport networks, including: standardized interfaces (Section II-A), architecture (Section II-B) and configuration (Section II-B) of photonic devices, signal impairments (Section III-F), performance aspects (Section II-E), and cost model (Section II-F). All elements presented throughout Section II define functional model of photonic network. Further, in Section III we define corresponding mathematical model associated with designing flows in photonic data transport network. Assumptions and construction of network graph are discussed in Sections III-A and III-B, respectively. Considered flow design problem is formulated as mixed-integer programme in Section III-C. Further, we investigate modeling specific aspects of the photonic data transport networks, related to: consistency of client flow at technology level (Section III-D), redundancy (Section III-E), impairments (Section III-F), and network cost (Section III-G). Paper is summarized with estimation of formulation complexity in Section IV, and conclusions in Section V.

\section{PhOTONIC DATA TRANSPORT NETWORKS}

Photonic data transmission exploits optical tracks (fibers) to send photonic signals between transceivers (lasers) and receivers (photo-diodes). Photonic signal is modulated to represent values of consecutive bits, composing transmitted piece of data. Low interference with other electromagnetic signals makes photonic signals stable and robust to distortions. Thus, photonic signals can be successfully sent over long distances, reaching thousands of kilometers, and transmitted messages can be still reproduced with small error rate. 
Photonic signals can be easily multiplexed in the frequency domain. Frequency in the case of photonic networking is called wavelength, and the corresponding multiplexing is called Wavelength Division Multiplexing or WDM. WDM is also a term describing the related channel-oriented transport technology, exploiting regular channel widths and spacing (socalled optical grid [1]). Each wavelength constitutes thus an isolated communication channel, in the following referred to as $\lambda$.

\section{A. Optical Transport Network}

G.709 [2] is an ITU-T recommendation defining interfaces of Optical Transport Network (OTN), defined in another ITU-T recommendation G.872 [3]. It characterizes so-called Optical Transport Hierarchy (OTH), i.e., structured hierarchy of interfaces that can be treated as an extension of the SDH hierarchy, defined in ITU-T recommendation G.707 [4]. Interfaces defined in G.709 are considered at two levels: user-to-network (UNI) and network-to-network (NNI). UNI interface is exposed towards an OTN client, and NNI interface is exposed towards an OTN network.

According to recommendation G.709, the basic OTH information structure is called Optical Channel (OCh). OCh spans between line ports of each pair of adjacent transponders, responsible for converting electrical data signal into photonic signal, modulated as single wavelength (and vice versa). Each OCh is associated with out-of-band signaling channel used for management and supervision of optical channel. OCh payload is filled with lower layer information structure called Optical Transport Unit (OTU). Similarly as OCh, OTU spans between two adjacent transponders, but it is associated with data connection in the electric domain. OTU signaling creates a control channel to exchange information related to supervision and conditioning signal for transport (including Forward Error Correction). Payload of OTU is further filled with information structure called Optical channel Data Unit (ODU). ODU is a function of termination point of digital end-to-end data path between two optical devices, crossing amplification and regeneration sections. ODU signaling is used to exchange control information related to end-to-end path supervision and monitoring so-called tandem connections.

Finally, client signal is mapped into information structure called Optical Payload Unit (OPU). OPU structure is responsible for the adaptation of the client signals and lower layer ODU signals. OPU overhead comprises information required to perform rate adaptation between the client signal rate and the OPU payload rate, and other OPU overheads supporting the client signal transport. OPU structure is filled into the ODU payload.

A number of the OCh structures is multiplexed into structure called Optical Multiplex Section (OMS). OMS is a function of termination point of the (de-)multiplexing section. OMS is further filled into the payload of structure called Optical Transport Section (OTS), responsible for transporting multiplexed optical channels between two adjacent amplification points through single fiber span. OTS is a function of termination point of

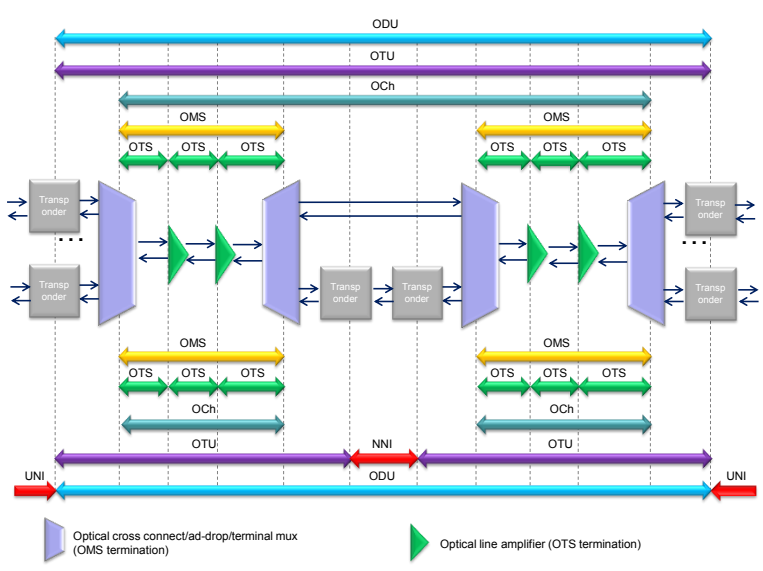

Fig. 1. OTN layers

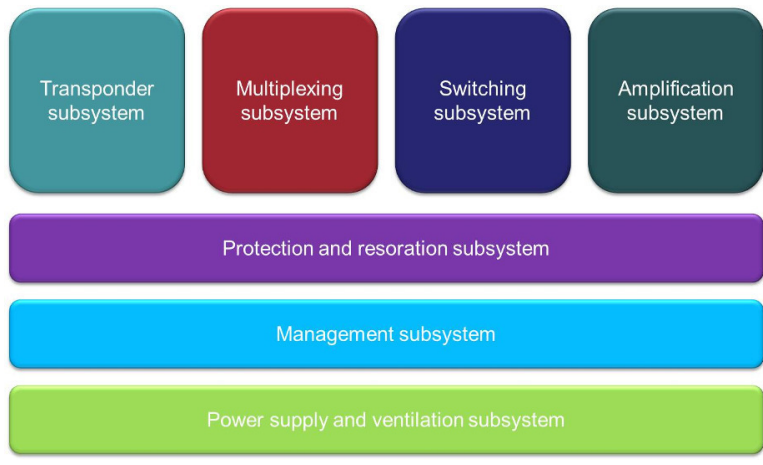

Fig. 2. ONE architecture

optical line which transports multiplexed optical channels from one node to another. OTN layers are presented in Figure 1. In the following, the introduced notation is used to map particular OTN functions into generic types of photonic cards needed to build OTN compatible networks.

\section{B. Architecture of optical network element}

Architecture of Optical Network Element (ONE) is composed of seven functional blocks presented in Figure 2. Each of these functional blocks is briefly described in the following.

1) Transponder subsystem: Transponder subsystem is a functional block responsible for adapting client black\&white signals, typically in $1310 \mathrm{~nm}$ band, to colorful $\lambda$ signals within $1550 \mathrm{~nm}$ band, according to used optical grid definition. Adaptation concerns both signal frequency and signal rate. Transponder is typically available in the form of expansion card, required for each add-drop channel. It provides UNI interface, and optionally NNI interface. Transponders are sometimes combined with Forward Error Correction blocks, that allow to correct some transmission errors, and decrease bit error rate.

Some vendors combine transponders with concatenation function, which allows to concentrate a number of low order speed signals into high order speed signal. For instance, four STM-16 (2.5Gbps) signals can be concentrated into one 


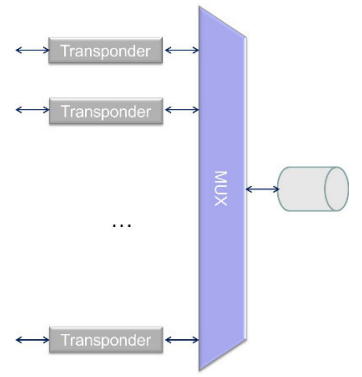

Fig. 3. Multiplexing subsystem: 1-stage multiplexer

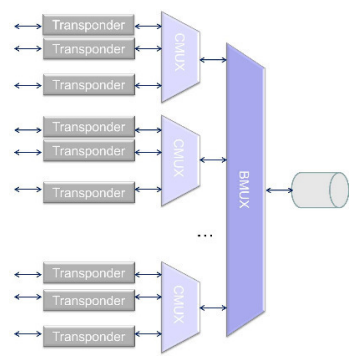

Fig. 4. Multiplexing subsystem: 2-stage multiplexer

ODU2 signal (10.709Gbps). Sometimes, concentrators are provided as stand-alone cards, further connected to transponder tributary interfaces. For instance, two GigabitEthernet signals (1Gbps each) using Generic Framing Procedure (GFP) can be concentrated into one STM-16 signal (2.5Gbps), further combined with mentioned $4 \times 2.5 G b p s$ transponder card. Such concentrating stand-alone card is called concentrator. Using concentrating function allows network operator to maintain fine grain granularity of transmitted signals, and optimized usage of the WDM network resources.

2) Multiplexing subsystem: Multiplexing subsystem is responsible for multiplexing multiple colorful add $\lambda$ signals produced by transponders into single multi-channel signal, according to definition of used optical grid. Similarly, demultiplexer separates particular drop channels from single multi-channel WDM signal. Thus, tributary side of multiplexing subsystem expose a number of physical interfaces towards transponder subsystem and single line interface towards switching subsystem.

For the sake of flexibility, vendors of optical devices often use multi-stage multiplexers. as those presented in figures 3 and 4. In 2-stage multiplexer, presented in Figure 4, multiplexing is decomposed in two stages: band multiplexing (BMUX) and channel multiplexing (CMUX). For instance, $N=80 \times \lambda$ loading plan, constructed according to $50 \mathrm{GHz}$ optical grid, can be decomposed into ten bands, each containing eight channels. Cost of 2-stage multiplexing with expandable CMUX cards can be more granular than 1-stage multiplexing. In particular, network operator may add new CMUX cards as its demand for capacity grows.

3) Switching subsystem: Switching subsystem is responsible for directing selected channels to the add-drop multi-

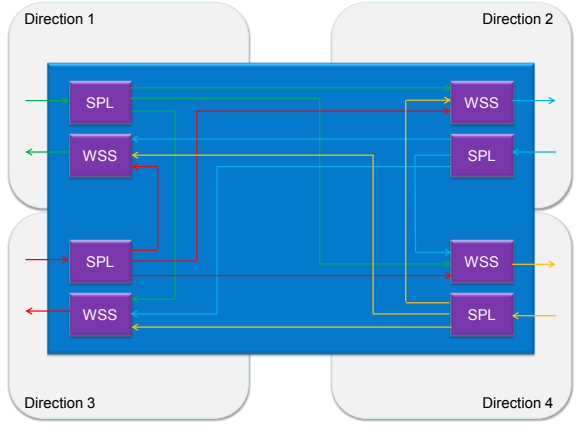

Fig. 5. Switching subsystem

plexing block and creating pass-through connections (between different ONE directions) for the other channels. In the passthrough mode, channel in one adjacent fiber is switched to channel in another adjacent fiber. Example 4-direction switching module is presented in Figure 5. Presented module is based on using Wavelength Selective Switches (WSS) and Optical SPlitters (SPL).

4) Amplification subsystem: Although optical signals do not interfere with other electromagnetic signals, power of optical signals is dispersed due to impurity of transmission medium. Thus, power of transmitted signal in the source node is much higher than power of received signal in the destination node. To assure right power level received by photo-detectors, optical signal may need to be amplified. Today, there are three types of optical amplifiers in common use:

- Semiconductor optical amplifier,

- Raman fiber amplifier,

- Erbium doped fiber amplifier.

Semiconductor Optical Amplifier (SOA), in a similar manner as Fabry-Perot laser diodes, uses a semiconductor to provide the gain medium. Signal amplification is realized in SOA by inducing energetic level of medium material. SOA amplifiers need anti-reflection components at the end faces, so amplifier not turns into a laser diode. SOA amplifiers in practice operate at signal wavelengths between $0.4 \mu \mathrm{m}$ and $2.0 \mu \mathrm{m}$, and generate gain up to $30 \mathrm{~dB}$. Compared with other amplifier types (mainly EDFA) SOA may generate higher noise, lower gain, moderate polarization dependence, and high non-linearity with fast transient time. Still, SOA amplifiers may have compact design, easily plug-able into fiber-pigtailed components. Despite relatively high noise level, high amplification and wide amplification spectrum make SOA amplifiers attractive option in certain optical applications. In particular, SOA amplifiers are widely used as wavelength convertors.

Raman Fiber Amplifier (RFA) exploits Raman distortion effect, i.e., nonlinear interaction between signal and pump laser within an optical fiber. In RFA amplifier, pump laser generates optical signal in lower range of optical spectrum $(1535 \mathrm{~nm})$ that is further coupled with operational signal in higher optical spectrum $(1540 \mathrm{~nm}-1580 \mathrm{~nm})$. In other words, RFA amplifier induces energy transfer from optical signal 
generated by pump laser to operational signal. RFA amplifier in practice generates low noise level, has high amplification level, and can produce high power signal. Construction of RFA amplfier may be relatively complex to provide smooth and wide gain characteristics, i.e., multiple pumping signals with different frequencies may be required.

Erbium Doped Fiber Amplifier (EDFA) is likely most widely used type of amplifiers. EDFA amplifier is composed of a short Erbium doped fiber span (typically several kilometers) and pump laser. Optical signal generated by the pump laser is absorbed by Erbium atoms, that in turn release absorbed energy to the operational signal transmitted through doped fiber. EDFA amplifiers in practical applications use $980 \mathrm{~nm}$ - 1480nm pumping signal to amplify operational signals transmitted at wavelength $1550 \mathrm{~nm}$. EDFA amplifiers in a process of Amplified Spontaneous Emission (ASE) generate noise that further decrease signal-to-noise ratio.

5) Protection subsystem: Typically, data stream, transmitted through WDM network, may be protected by parallel optical channel spreading between the same type of transponders in the same end devices as the nominal channel. For this purpose, vendors provide special protection cards responsible for activating protection channel in case of unavailability of the nominal channel. Such cards can work in one of two commonly used modes:

- source switched,

- destination switched.

Source switch mode is realized by turning off laser in the nominal channel, and immediate activation of laser in the protection channel, as only failure is detected. Source switch is thus completely transparent to carried data, and directly switches optical signals. Besides, source switch mode allows to use simple optical couplers at receiver side, because only one signal: nominal or protection is transmitted at time.

Destination switch is realized as data switch at receiver side. Switch continuously compares quality of nominal and protection signals, and according to result of this comparison passes one of them to client interface. It means that lasers of both signals must be active all the time, and data stream must be replicated to both channels at source.

6) Management subsystem: According to OTH hierarchy, overhead information can be sent in-band only to certain layer defined by OTU. Below OTU layer, signaling is sent out-ofband through so-called Optical Supervisory Channel (OSC). OSC organization is proprietary.

\section{ONE configuration}

Basic element of the WDM network is device called Optical Add-Drop Multiplexer (OADM). In general, OADM is responsible for multiplexing and concatenating tributary signals into line signals transmitted towards other devices. Depending on its configuration and design, optical devices can be used in different fashions. Typical optical device configurations include:

1) Line terminal (LT),

2) Back-to-back (BtB),

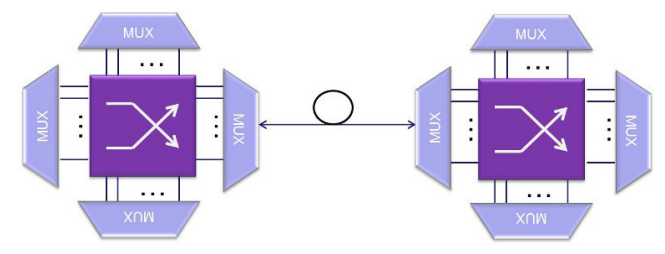

Fig. 6. Opaque mode

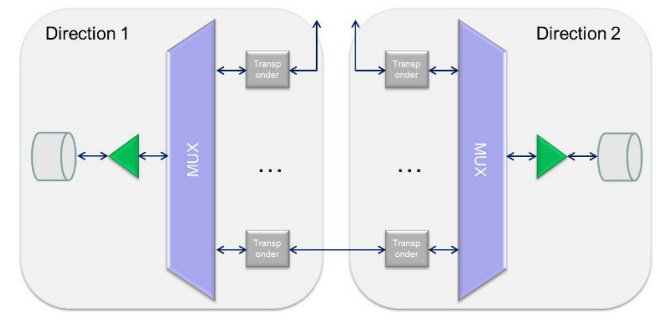

Fig. 7. Back-to-back configuration

3) Band optical add-drop multiplexer (BOADM) - 2-stage multiplexer only,

4) Reconfigurable optical add-drop multiplexer (ROADM),

5) Tunable and reconfigurable optical add-drop multiplexer (T\&ROADM).

Below, we briefly characterize each of these configurations.

1. Line terminal is the most basic configuration of an optical device. Device configured as a line terminal is responsible for adopting and modulating tributary signals as colorful signals and multiplexing resulting signals into $\lambda$ channels according to selected optical grid definition. Line terminals are commonly used in so-called opaque mode (see Figure 6), to build simple point-to-point optical spans. In opaque mode, device receiving optical signal transforms it to the electric domain and switches obtained electrical signals. Opaque mode is common configuration of the SDH/Sonet devices. Line terminal can be equipped with transponders, concentrators, multiplexers, and amplifiers.

2. Back-to-back configuration allows to create simple multipoint optical networks, by static coupling tributary ports in transponders. Back-to-back configuration is presented in Figure 7.

3. Band optical add-drop multiplexer is an extension of back-to-back configuration which additionally allows subset of band signals to be transmitted in pass-through mode directly between band multiplexers (BOADM configuration, presented in Figure 8, refers to 2-stage add-drop multiplexers.)

4. Reconfigurable optical add-drop multiplexer is a configuration of optical device capable to add, drop, and passthrough wavelengths (switch between adjacent fibers). It can be (remotely) configured (through management system) to put each channel within line fiber into one of two states: passthrough or add-drop (see Figure 9).

5. Tunable and reconfigurable optical add-drop multiplexer is the most advanced configuration that extends remote configuration of ROADM with wavelength conversion function. 


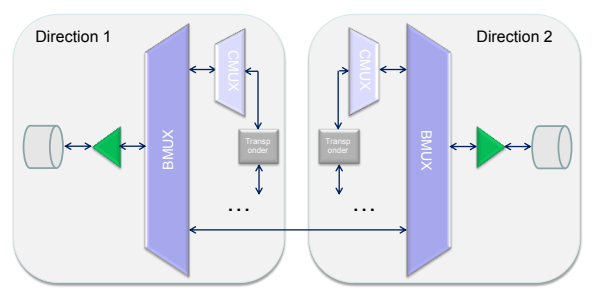

Fig. 8. Band optical add-drop multiplexer configuration

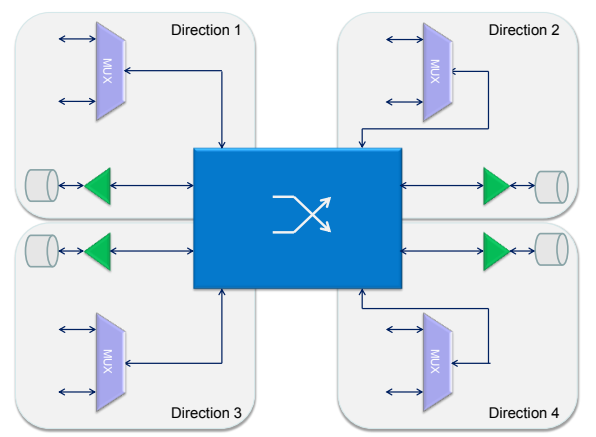

Fig. 9. Remote optical add-drop multiplexer configuration

\section{Optical impairments}

Fiber attenuation is the most fundamental impairment that affects optical signal propagation. Attenuation is a fiber property resulting from using various material, structural, and modular impairments. Still, fiber attenuation is an effect with intensity linearly proportional to fiber length and number of optical elements: connectors, splitters, etc. Thus, optical signal power lost due to fiber attenuation can be recovered by using so-called Linearized Optical Fiber Amplifier (LOFA) cards, containing amplification modules described in Section II-B4. Beyond amplifiers itself, LOFA cards may also contain a module called Variable Optical Attenuator (VOA), responsible for enforced attenuation of optical signal, so volume of power received by photo-diode is contained in strictly defined working window. Attenuation of VOA modules is typically dynamically set in a closed-loop feedback between adjacent ONE's. Unfortunately, amplifiers, as all active optical elements, introduce into transmitted signal some portion of noise. Fiber attenuation is well standardized for particular fiber types (cf. [5]).

Further, shape of optical signal can be distorted due to dispersion. Dispersion cause that optical impulse is widened in time. In extreme case, two consecutive impulses can overlap, leading to potential reception errors. Two types of dispersion ca be categorized: chromatic dispersion and polarization mode dispersion.

Chromatic dispersion is related to velocity difference be- tween different wavelengths in particular medium. Due to linear character of chromatic dispersion, impairments introduced by this effect can be eliminated by using fiber spans with reverse dispersion characteristics. Compensating modules, called Dispersion Compensating Fiber (DCF), are typically attached after each fixed-length section of long-haul connection.

Polarization mode dispersion is caused by asymmetry of the fiber-optic strand. Polarization mode dispersion has thus completely random character. According to ITU-T recommendation G.652 [5], unit dispersion coefficient of optical fiber G.652B should not cross $17 \mathrm{ps} /(\mathrm{nm} \times \mathrm{km})$ for chromatic dispersion and $0.20 \mathrm{ps} /(\sqrt{\mathrm{km}})$ for polarization mode dispersion.

Another serious source of impairments is light scattering. Light scattering results from localized non-uniformity in the fiber medium. It can be seen as a deflection of a ray from a straight path. Deviations from the law of reflection due to irregularities on a surface of optical connectors are also usually considered to be a form of scattering. Among light scattering effects, several effects can imply serious impairments in photonic networks: stimulated Brillouin scattering and stimulated Raman scattering. Scattering effects are non-linear, and they tend to manifest themselves when optical signal power is high.

Other serious non-linear impairments in photonic networks are related to: Four-Wave Mixing, Self-Phase Modulation, and Cross-Phase Modulation. These effects result from transmitting multiple wavelengths over single fiber. Non-linear effects increase level of noise in the optical signal.

\section{E. Network performance}

During transmission in medium, optical signals are distorted by certain optical effects. Resulting transmission errors affects OTN service quality. Some of effects, having linear characteristics, like fiber attenuation or chromatic dispersion, can be compensated by using dedicated modules. Other effects introduce distortions that cannot be recovered without translation to electric domain.

Major performance indicator of OTN service quality, called Bit Error Rate or simply BER, is thus related to the number of erroneously transmitted bits in digital client signal. In relation to noise introduced by non-linear optical effects and active optical elements, BER can be treated as a function of Optical Signal to Noise Ratio (OSNR), representing gap between power of optical signal and power of noise. In relation to effects not changing power level, but its distribution in time, BER can be treated as a function of dispersion coefficients.

OSNR related to sequence of active elements can be calculated according to formula (1).

$$
1 / O S N R=\sum_{i} 1 / O S N R_{i},
$$

where $O S N R_{i}$ determines partial OSNR of $i$-th element in the sequence.

\section{F. Network cost model}

Adjacent optical devices are connected by optical fibers, attached to their line interfaces. Multiple fibers are further combined into optical cables, connecting network sites and 
wells. Cables are attached to optical distribution frames, where incoming fibers are interconnected in order to establish end-toend optical spans between devices. Signaling between WDM devices is realized via proprietary out-of-band channel, outside optical grid.

Optical device is a complex device, equipped with variety of specialized functions required for photonic signal processing. Typically, those specialized functions are realized by expansion cards, fitted into device slots. Cards can be further interconnected by back-plane device wiring or through external patch-cords, fitted manually into their front panel interfaces. In practice, to transport client signals, optical device must be equipped with a minimal set of supervisory cards, responsible for device control and management. Those cards implement proprietary vendor-specific algorithms to enable proper transmission and reception of photonic signals between adjacent devices. In the following, cost related to those cards is treated as a part of device fixed cost, being in turn a part of CAPital EXpenditure (CAPEX) related to network deployment.

In practice, optical devices are sold in the form of racks (for example, one rack per direction), equipped with certain number of slots for shelves. Each shelf in turn may be equipped with a number of slots to host expansion cards. Tributary and linear cards may be combined with embedded or external Network Interface Controllers (NICs). In the latter case, external NICs are fitted into appropriate slots on cards. ONE slots must thus be filled with a set of required expansion cards providing specialized functionality. Expansion cards can be classified into two categories:

- fixed cards: switching matrices, band multiplexers, supervision cards, fans, power suppliers, etc.,

- elastic cards: channel multiplexers, transponders, and muxponders.

Number and type of fixed cards is predefined by system vendor. Cost of fixed cards, together with cost of racks and shelves, is accounted into system installation cost. In other words, cost of the fixed cards is independent of traffic amount handled by ONE. Contrary to fixed cards, number and type of elastic cards can vary according to specific usage of the WDM system. In particular, network operator may choose between different types of transponders providing different modulation types, capacity, and forward error correction methods. In some ONE designs, channel multiplexers can be not expandable, and full range of multiplexers must be installed in the form of fixed cards. However, in the following, we consider elastic channel multiplexer cards as more general case. Band multiplexers are typically fixed cards. Still, due to particular composition of the switching matrices, some ONE designs may require installation of one rack per each direction (representing long-haul connection with neighboring ONE). In that case, predefined set of fixed cards, including band multiplexers, must be installed in each rack.

According to ITU-T recommendation G.709 [2], signals transmitted through OTN network compose the OTH hierarchy. OTH also defines structure and bandwidth of tributary signals crossing the UNI interface of OTN network. As bandwidth of single WDM channel may be greater than bandwidth of typically used Layer 2 signals, multiple client signals may be concatenated into larger signals, better fitted to bandwidth of WDM channels. Signals are typically concatenated in the time domain through dedicated concentrator cards.

Tributary signals need to be further framed according to ODU definition, coupled with error correction overhead, and transformed into colored optical signals to be transmitted through WDM network. Card responsible for these functions is called transponder. Optical signal transmitted by source transponder is again converted to the digital domain in the destination transponder. Between transponders, signal remains in the optical domain. Still, certain control information related to the transmitted signal is carried along signal path through dedicated administration channel.

\section{FLOW DESIGN}

In this section we consider flow design problem related to OTN/WDM photonic networks. Considered flow design problem is formulated in terms of mathematical programming. Having given basic WDM network topology and set of traffic demands to be realized, OTN/WDM flow design problem is aimed at identifying a flow distribution and composition of expandable WDM components (e.g., muxponders, transponders, multiplexers, etc.) leading to optimized value of certain objective function. In particular, feasible solution of the considered problem identifies design of the ONE nodes in terms of number, type, and configuration of expansion cards necessary to realize traffic demands, and associated cost.

Considered problem is described in the literature as Routing Wavelength Assignment (RWA) problem. It is commonly considered in combination with objective function maximizing the number of concurrent connections. Example integer linear programming formulation of this problem can be found in [6]. Independently in [7] and [8] it was proved that RWA problem is $\mathcal{N} \mathcal{P}$-complete. Formulations proposed in the literature ([9], [10], [11], [12], [13], [14], [15], [16], [17], [18], [19], [20]) differ from formulation proposed in the following in terms of graph construction. Namely, in this paper it is assumed that each $\lambda$ channel constitutes separate edge in the network graph. Such assumption is not common in other works, but it allows to simplify formulation, and increase problem flexibility through graph construction. Moreover, classical RWA problem is concerned with routing and $\lambda$ selection only. Here, problem is extended with consideration of the access side. This extension is motivated by usage of objective function related to cost of elastic expansion cards.

\section{A. Assumptions}

Traffic demands are assumed to be known in advance, e.g., they can be sourced from some external business forecast and measurement tool. As demand variation is out of the scope at considered network design problem, demand volumes may be additionally adjusted with some security margin. Each traffic demand is defined by triple: source, destination, and bandwidth 
volume. Demand source and destination are external clients connected to local ONE nodes through intra-office or shorthaul black\&white fibers.

Client devices and ONE devices are installed within Points of Presence (PoPs) of a network operator, and each client device is connected to uniquely defined ONE, usually in the same PoP. Even if in some PoP, ONE device is not installed, client localized in such PoP must be unambiguously assigned and connected to one ONE in one of the other PoPs.

After installing full suite of channel multiplexers, ONE device is capable to handle $N$ channels, equal to its maximum capacity. Each channel has precisely defined central frequency and width. Central frequencies of consecutive channels are supposed to be compatible with one of optical grids defined by ITU-T.

\section{B. Network graph}

Network topology at the simplest level defines locations, configuration, and type of network elements, and arrangement of long-haul fibers connecting network elements. Depending on required level of granularity, network topology can be more or less detailed. At level of details required by flow optimization, this simple topology needs to be extended with deeper insight into composition of network elements. For this purpose, we define a directed graph $\mathcal{G}(\mathcal{V}, \mathcal{E})$ composed of set of nodes $\mathcal{V}$ and set of edges $\mathcal{E}$. Graph composition is used in the following as a basic modeling methodology. It allows to formulate considered flow design problem in terms of multi-commodity flow optimization.

1) Graph nodes: In general, node set $\mathcal{V}$ can refer to four types of physical elements (cards or whole devices):

- optical network element - device responsible for multiplexing, switching, and (optionally) converting colorful $\lambda$ signals $(\mathcal{O})$,

- transponder - expansion card responsible for adopting colorless tributary signals and modulating them as colorful $\lambda$ signals $(\mathcal{T})$,

- muxponders - expansion card responsible for concatenating multiple colorless signals into higher-order colorful signals $(\mathcal{M})$,

- client - non-WDM device, consuming OTN services $(\mathcal{C})$.

In order to model switching and converting colorful $\lambda$ signals, each ONE is represented in the network graph $\mathcal{G}$ by a set of graph nodes (referred to as colorful nodes), each associated with exactly one $\lambda$ and one direction towards adjacent ONE. Accordingly, number of colorful graph nodes associated with single $\mathrm{ONE}$ is equal to $N \times D$, where $D$ is the number of ONE neighbors. Similarly, basic graph of long-haul fiber connections is replicated, so there exists $N$ (equal to number of $\lambda$ 's) parallel subgraphs, each topologically isomorphic with original network graph. If ONEs are capable to convert $\lambda$ frequencies, all colorful nodes associated with single ONE needs to be interconnected. For example, if in the feasible solution, such artificial link is crossed on path between colorful nodes associated with $\lambda_{1}$ and $\lambda_{2}$, it means that signal

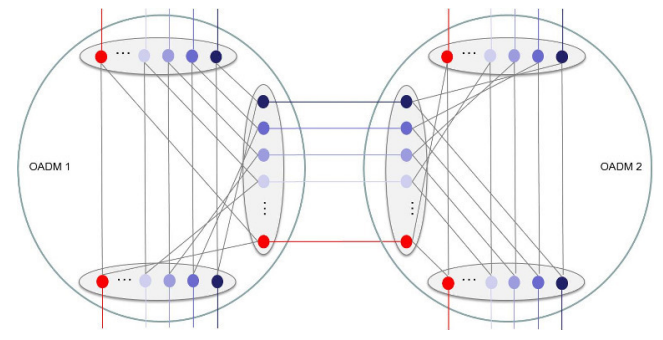

Fig. 10. ROADM subgraph

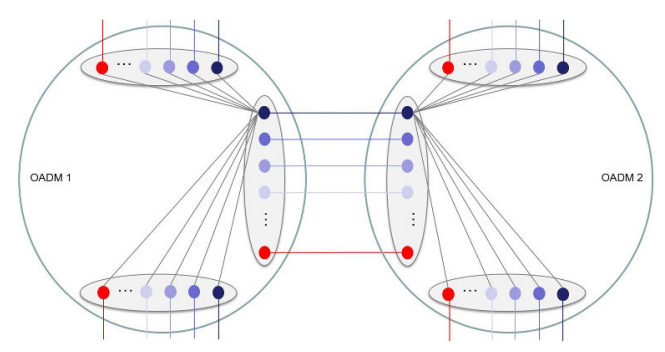

Fig. 11. T\&ROADM subgraph

incoming to the related ONE at $\lambda_{1}$ is transmitted out through $\lambda_{2}$. Example subgraph of colorful nodes associated with two neighboring 3-direction ONEs in ROADM configuration is presented in Figure 10. T\&ROADM counterpart extends the ROADM subgraph with full mesh connections between colorful nodes inside ONE, as presented in Figure 11.

Transponder and concentrator are sometimes combined as one expansion card - muxponder. If not combined, clients can be connected to transponders two-fold: through direct connections or indirectly through hierarchy of compatible concentrators. In the network graph, stand-alone transponders are associated with a subset of graph nodes $\mathcal{T}$, where each graph node is associated with one transponder type and one ONE location. Associated subgraph is presented in Figure 12. In the figure, there are three transponder types (say 10Gbps, 40Gbps, and 100Gbps) and two clients. Transponder graph nodes representing each transponder type in one location are connected to all colorful nodes.

Muxponders form subset of graph nodes $\mathcal{M}$, where each muxponder type is replicated $N$ times, so each colorful node

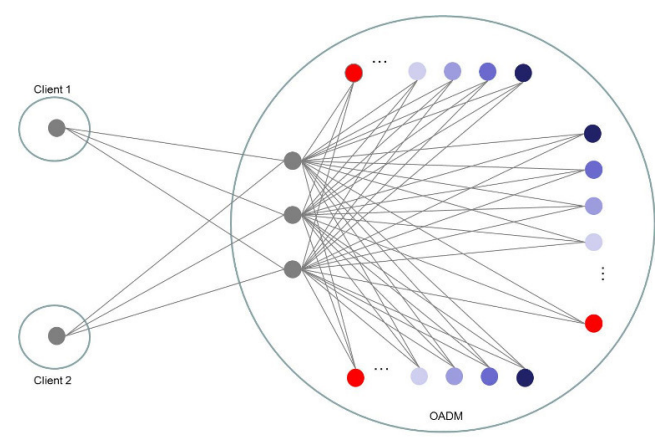

Fig. 12. Transponder subgraph 


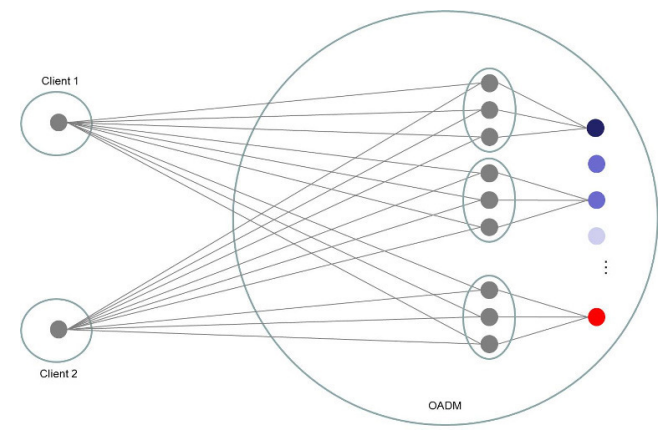

Fig. 13. Muxponder subgraph

can be connected to its own unique suite of muxponders. Despite graph contains all potential muxponder cards, only some subset of cards may be required in the optimal solution. All muxponder graph nodes are connected with graph nodes representing compatible interfaces in the client devices, as it is presented in Figure 13. Whole muxponder hierarchy is represented by single graph node, which means that multiple different connection types (different transport technology modules) are represented as parallel graph links, each associated with one transport technology type.

Client devices are not replicated in the network graph, and there exists exactly one graph node associated with each physical client device.

2) Graph edges: Nodes $\mathcal{V}$ are connected by set of edges $\mathcal{F}$, referring to physical connections:

- long-haul fibers connecting ONEs $(\mathcal{H})$,

- intra-office fibers connecting line ports in client devices and tributary ports in muxponders/transponders,

- patch-cords and back-plane wiring connecting line ports in transponders and tributary ports in ONE multiplexers.

All enumerated types of connections: fibers, patch-cords, and wiring are further described by common term link. Set $\mathcal{F}$ is assumed to be a superset of the link set defined by the basic network topology. In particular, it contains replicated edges between adjacent colorful nodes. Finally, not all edges contained in $\mathcal{F}$ will be deployed, because some graph nodes represent non-existent components and link deployment will depend on card installation. A subset of these potential links will be selected for deployment or activation.

\section{Mathematical formulation}

Based on introduced network graph definition, the WDM flow design problem can be formulated as below mixed integer programme.

object sets

$\mathcal{E}$
$\mathcal{V}$
$\mathcal{O} \subset \mathcal{V}$
$\mathcal{T} \subset \mathcal{V}$
$\mathcal{M} \subset \mathcal{V}$
$\mathcal{C} \subset \mathcal{V}$
$\mathcal{F}=\mathcal{H} \cup \mathcal{L}$
$\mathcal{G} \subset \mathcal{F}$
$\mathcal{H} \subset \mathcal{F}$
$\mathcal{L} \subset \mathcal{F}$

$\mathcal{A}_{v} \subset \mathcal{F}$

$\mathcal{B}_{v} \subset \mathcal{F}$

$\mathcal{P}_{v} \subset \mathcal{F}$

$\mathcal{Q}$

predefined objects

$a(e) \in \mathcal{C}$ originating client node (source) of

$b(e) \in \mathcal{C} \quad$ terminating client node (sink) of de-

$a(f) \in \mathcal{V}$

$b(f) \in \mathcal{V}$

$\alpha(f e) \in \mathcal{L}$

\section{constants}

$c_{e}$
$l_{f}$
$t_{v}$

$l_{f}$

$n_{f}$

variables

$s_{f e} \in\{0,1\} \quad$ variable equal to 1 if demand $e \in \mathcal{E}$ is realized on link $f \in \mathcal{F}$, and 0 otherwise

$z_{f} \in \mathbb{Z} \quad$ variable equal to the number of transport modules on link $f \in \mathcal{F}$

\section{constraints}

$$
\begin{array}{ll}
\sum_{f \in \mathcal{A}_{v}} l_{f} s_{f e}=c_{e} & e \in \mathcal{E}, v=a(e) \in \mathcal{C} \\
\sum_{f \in \mathcal{B}_{v}} l_{f} s_{f e}=c_{e} & e \in \mathcal{E}, v=b(e) \in \mathcal{C} \\
\sum_{f \in \mathcal{A}_{v}} s_{f e}=\sum_{f \in \mathcal{B}_{v}} s_{f e} & e \in \mathcal{E}, v \in \mathcal{V} \backslash\{a(e), b(e)\}(2 \mathrm{c}) \\
\sum_{e \in \mathcal{E}} s_{f e} \leq z_{f} & f \in \mathcal{L} \\
\sum_{e \in \mathcal{E}} s_{f e} \leq M z_{f} & f \in \mathcal{H} \\
\sum_{f \in \mathcal{A}_{v}} z_{f}=\sum_{f \in \mathcal{B}_{v}} z_{f} & v \in \mathcal{O} \\
\sum_{f \in \mathcal{B}_{v}} z_{f} \leq t_{v} & v \in \mathcal{M} \cup \mathcal{O}
\end{array}
$$




$$
\begin{array}{ll}
\sum_{f \in \mathcal{A}_{v}} z_{f} \leq 1 & v \in \mathcal{M} \cup \mathcal{O} \\
z_{f} \leq n_{f} & f \in \mathcal{F} .
\end{array}
$$

Presented formulation is a modified form of classical formulation of multi-commodity flow optimization problem (see [21] and [22]). In this formulation, flow distribution is described by values of binary variables $s$ representing flows on particular network links. Having given feasible values of $s$ one can easily reconstruct particular paths selected to carry traffic.

Integer variables $\boldsymbol{z}$ determine in general number of transmission modules on particular links. However, in case of links associated with nodes $v \in \mathcal{M} \cup \mathcal{O}$ this number is strictly binary (due to constraints (2h) and (2i)). For the rest, variable $z$ is integer (due to constraints $(2 \mathrm{~g})$ and (2i)).

Due to classical flow conservation constraints (see [21]), in relation to specific demand, in all nodes, except end nodes of this demand, the total volume of incoming flows must be balanced by total volume of outgoing flows. Formulation (2) involves two groups of flow conservation constrains: constraints (2a)-(2c) related to variables $s$ and constraints (2f) related to variables $\boldsymbol{z}$.

Usage of particular network links, including all types of inter-card patch-cords and back-plane wiring, by flows determines consumption of transport modules (their number is expressed by variables $\boldsymbol{z}$ ), according to constraints (2d) and (2e).

Constraints $(2 \mathrm{~g})$ assure that only one transponder or muxponder can be coupled with each channel tributary port in ONE multiplexer. Similarly, number of active tributary and line links connected to muxponder ports are limited by constraints $(2 \mathrm{~g})$ and $(2 \mathrm{~h})$, respectively.

Formulation (2) gathers constraints related to using WDM transport to carry client traffic. Based on this formulation, in the following we consider a number of its extensions and composition of objective function related to the overall cost associated with WDM transport.

\section{L2 technology}

To express that each demand can be realized using homogenous L2 technology, like GigabitEthernet, FC800, STM64, binary variable vector $\boldsymbol{k}$ was introduced. Non-zero value of variable $k_{g e}$ enforces through constraints (3a) that demand $e \in \mathcal{E}$ can be realized using only links compliant with technology $g \in \mathcal{Q}$. If one technology (say $g \in \mathcal{Q}$ ) is selected (value $k_{g e}$ is 1 ), links associated with other technologies cannot be used, what is assured by constrains (3b).

$$
\begin{array}{ll}
\sum_{f \in \mathcal{R}_{g}} s_{f e} \leq\left|\mathcal{R}_{g}\right| k_{g e} & g \in \mathcal{Q}, e \in \mathcal{E} \\
\sum_{g \in \mathcal{Q}} k_{g e} \leq 1 & e \in \mathcal{E} .
\end{array}
$$

Above, $\mathcal{R}_{g} \subset \mathcal{F}$ denotes set of edges associated with technology $g \in \mathcal{Q}$.

\section{E. Redundancy}

To provide uninterrupted services, able to survive failures of optical network elements and fiber connections, client devices need additional bandwidth, allocated along paths not affected by considered failures. Additional bandwidth, required by protection, is associated with certain level of resource redundancy. Redundant resources are either not used in the nominal network state or can be used for transmitting low priority traffic, preempted in case of failure occurrence.

In case of the WDM networks, redundant resources can be provided either at client digital signal level (called client protection) or photonic signal level (called photonic protection). In the former case, client device is responsible for activating redundant resources. Redundant resources cover optical channels allocated along protection path, and transponder/muxponder cards and ports. In the latter case, specialized protection cards are required. Such protection cards split power of the protected optical signal between multiple (usually two) ports connected to different add-drop ports within multiplexer subsystem. In both cases, the nominal and protection paths should be topologically disjoint with regard to failure occurrence, so under any failure at least one of the paths survives.

Let set $\mathcal{F}_{i}, i \in \mathcal{I}$ represents an arbitrary set of links that share risk of failure. Such group is described in the literature as Shared Risk Link Group (SRLG) or in general Shared Risk Resource Group (SRRG) [23]. Each SRLG associated with single link $f \in \mathcal{F}$ failure contains exactly one element. Each SRLG associated with node $v \in \mathcal{O}$ failure contains all adjacent links, i.e., $\mathcal{A}_{v} \cup \mathcal{B}_{v}$. SRLG should be constructed case by case in relation to specific needs and requirements of a network operator. Specific composition of SRLG thus remains out of scope of this paper.

In order to determine capacity $c_{e i}$ allocated to demand $e$ and available during failure state $i$, constraints (4a)-(4b) should be added to the problem formulation (2):

$$
\begin{array}{lll}
0 \leq c_{e}-c_{e i} \leq M r_{e i} & e \in \mathcal{E}, i \in \mathcal{I} \\
0 \leq c_{e i} \leq M\left(1-r_{e i}\right) & e \in \mathcal{E}, i \in \mathcal{I} \\
0 \leq r_{e i} \leq \sum_{f \in \mathcal{F}_{i}} s_{f e} \leq M r_{e i} & & e \in \mathcal{E}, i \in \mathcal{I} .
\end{array}
$$

Above, each variable $c_{e i}$ expresses volume of link flow associated with demand $e$ in failure state $i$. Value of variable $r_{e i}$ indicates if link $e$ is available throughout failure state $i$. Consequently, if for some pair $(e, i) r_{e i}=1$ then associated $c_{e i}$ is equal to $c_{e}$, and $c_{e i}$ is zero otherwise. In according to constraints (4c), value of $r_{e i}$ is positive if and only if at least one link $f$ realizing demand $e$ is affected by failure $i$, i.e., when $\sum_{f \in \mathcal{F}_{i}} s_{f e} \geq 0$.

Redundancy required by protection mechanisms can be also modeled through multiplication of demand volume to be realized by the transport WDM/OTN network, and additional constraints assuring that only fraction of demand volume is transmitted through specific resources (network element or link). Protection method associated with described resource redundancy requirement is commonly described in the literature as path diversity [24]. To assure introduced requirement constraints (2a)-(2b) must be rewritten as: 


$$
\begin{aligned}
& \sum_{f \in \mathcal{A}_{v}} l_{f} s_{f e}=2 c_{e} \\
& e \in \mathcal{E}, v=a(e) \\
& e \in \mathcal{E}, v=b(e) \\
& e \in \mathcal{E}, i \in \mathcal{I} \text {. }
\end{aligned}
$$

Constraints $(5 \mathrm{c})$ assure that any link flow do not exceed demand volume. In result, each demand must be realized on at least two disjoint paths.

\section{F. Optical impairments}

Optical impairments (described in Section III-F) affecting optical signals, transmitted through photonic network, can be modeled in the form of three limitations:

- fiber length limit,

- hop-count limit,

- noise accumulation limit.

Chromatic dispersion is responsible for spreading duration of optical signal peaks. As a linear effect, proportional to the total length of fiber, chromatic dispersion can be eliminated by using DCM modules. DCM modules are supposed to introduced chromatic dispersion in reverse direction than dispersion introduced by regular fiber. DCM modules compensate thus chromatic dispersion related to central frequency of the optical signal. Consequently, dispersion affecting frequencies far from the central frequency are not compensated completely. Amount of uncompensated chromatic dispersion is called residual dispersion. Residual dispersion is an important factor that limits the total length of fiber traversed by optical signal. Maximum admissible fiber length depends on transponder type and characteristics.

Optical signal propagating through fiber medium is attenuated. Fiber attenuation is proportional to the total length of fiber spans crossed by the signal. To restore signal power to the level required by photo-detector, optical signal is amplified by LOFA cards localized in selected points along the path. However, LOFA cards, beside signal amplification, introduce some portion of noise. To keep signal quality at high level, network operator should control the total amount of introduced noise. On one hand, low noise level can be assured by hop-count constraints. On the other hand, noise characteristic of active optical elements, as mentioned LOFA cards, can be expressed in terms of ONSR value. As described in Section II-E, total value of OSNR is proportional to partial OSNR of particular elements in the path.

All introduced in this section limitations can be associated with additive metrics: length, hop-count, and inverse OSNR. Accordingly, all can be modeled similarly by set of so-called shortest path constraints. Formulation of shortest path constraints is based on path length variables $\boldsymbol{p}=\left(p_{v}: v \in \mathcal{O}\right)$. Each $p_{v}$ represents the length of the shortest path from $v$ with respect to weight system $\boldsymbol{q}$. Then, for each link $f$ outgoing from node $v(a(f)=v)$ contained in the shortest path crossed by edge the following shortest path condition must hold.

$$
p_{a(f)}+q_{f}=p_{b(f)} .
$$

Condition (6) is commonly used by the shortest path algorithms to validate if the path traversing edge $f$ is shorter than the shortest path found so far. For our purposes we adopt condition (6) to formulate shortest path constraints:

$$
\begin{aligned}
& p_{a(f)}+q_{f}-p_{b(f)}=0 \text { if value of } z_{f} \text { is } 1 \quad f \in \mathcal{E} \quad \text { (7a) } \\
& p_{a(f)}+q_{f}-p_{b(f)} \geq m \text { if value of } z_{f} \text { is } 0 \quad f \in \mathcal{E} \text {. (7b) }
\end{aligned}
$$

Conditions (7a)-(7b) state that if and only if edge $f$ is contained in the shortest path to $b(f)$, length of this path must be equal to sum of the length of a shortest path to $a(f)$ and weight $q_{f}$. Otherwise; the value of the expression $p_{a(f)}+q_{f}-p_{b(f)}$ must be greater or equal to $m$, which is the smallest difference between lengths of two paths. Accordingly, length limitation constraints can be formulated as follows:

$$
\begin{array}{ll}
m\left(1-z_{f}\right) \leq p_{a(f)}+q_{f}-p_{b(f)} \leq M z_{f} & f \in \mathcal{E} \\
p_{v} \leq p^{*} & v \in \mathcal{V} .
\end{array}
$$

Considered limitations require additional constraints $(8 \mathrm{~b})$ to enforce that values of required parameters remain under maximum admissible level $p^{*}$. Weight system $\boldsymbol{q}$ is constant, and is supposed to express value of required parameter:

- link length,

- number of active elements associated with link (usually one),

- inverse OSNR associated with link.

\section{G. Network cost}

Cost of WDM transport is mostly related to the number and type of used elastic expansion cards: channel multiplexers, transponders, and muxponders. Cost related to installation of transponder and muxponder cards can be expressed as follows:

$$
\sum_{v \in \mathcal{O}} \sum_{f \in \mathcal{P}_{v}} \frac{1}{2} g_{v} z_{f}
$$

Above, unitary cost related to card associated with node $v \in \mathcal{M} \cup \mathcal{T}$ is given by constant $g_{v}$.

To calculate cost related to installation of multi-stage multiplexer expansion cards we need to introduce additional variables and constrains. Binary variable $m_{j}$ associated with multiplexer $j \in \mathcal{J}$ states if card is installed or not. Variable is positive if at least one channel associated with this particular multiplexer is used. This relation is expressed by constrains (10). Set of colorful channel links and cost associated with multiplexer $j \in \mathcal{J}$ are given by $\mathcal{S}_{j}$ and $h_{j}$, respectively.

$$
\sum_{f \in \mathcal{S}_{j}} z_{f} \leq\left|\mathcal{S}_{j}\right| m_{j} \quad j \in \mathcal{J}
$$

Finally, with respect to (10) and other constraints defined above, the objective function can be formulated as:

$$
\min F(\boldsymbol{z}, \boldsymbol{m})=\sum_{v \in \mathcal{O}} \sum_{f \in \mathcal{P}_{v}} \frac{1}{2} g_{v} z_{f}+\sum_{j \in \mathcal{J}} h_{j} m_{j}
$$

Objective function (11) is related to minimization of number of expansion cards. 
TABLE I

CHARACTERISTICS OF THE SELECTED NETWORK INSTANCES

\begin{tabular}{|l|c|c|c|}
\hline Network instance & Nodes & Links & Demands \\
\hline abilene & 12 & 15 & 132 \\
\hline atlanta & 15 & 22 & 210 \\
\hline brain & 161 & 332 & 14311 \\
\hline cost266 & 37 & 57 & 1332 \\
\hline geant & 22 & 36 & 462 \\
\hline germany50 & 50 & 88 & 662 \\
\hline giul39 & 39 & 172 & 1471 \\
\hline france & 25 & 45 & 300 \\
\hline janos-us & 26 & 84 & 650 \\
\hline janos-us-ca & 39 & 122 & 1482 \\
\hline
\end{tabular}

TABLE II

CHARACTERISTICS OF THE NETWORK GRAPHS

\begin{tabular}{|l|c|c|c|c|c|}
\hline Network instance & $|\mathcal{O}|$ & $|\mathcal{C}|$ & $|\mathcal{L}|$ & $|\mathcal{H}|$ & $|\mathcal{F}|$ \\
\hline abilene & 2400 & 12 & 1200 & 36 & 1236 \\
\hline atlanta & 3520 & 15 & 1760 & 45 & 1805 \\
\hline brain & 53120 & 161 & 26560 & 483 & 27043 \\
\hline cost266 & 9120 & 37 & 4560 & 111 & 4671 \\
\hline geant & 5760 & 22 & 2880 & 66 & 2946 \\
\hline germany50 & 14080 & 50 & 7040 & 150 & 7190 \\
\hline giul39 & 27520 & 39 & 13760 & 117 & 13877 \\
\hline france & 7200 & 25 & 3600 & 75 & 3675 \\
\hline janos-us & 13440 & 26 & 6720 & 78 & 6798 \\
\hline janos-us-ca & 19520 & 39 & 9760 & 117 & 9877 \\
\hline
\end{tabular}

\section{COMPLEXITY}

Throughout this section we estimate complexity of the considered formulation of the WDM flow design problem. Complexity estimation is based on calculation of the numbers of variables and constrains necessary to formulate the considered WDM flow design problem in relation to the selected instances of network instances defined in the SNDLib library [25]. Referenced network instances are characterized in Table I, where particular columns contain the numbers of network nodes, network links, and traffic demands, respectively.

Further, assuming 80-channel WDM technology and three types of transponders (10Gbps, 40Gbps, 100Gbps) we calculate the numbers of particular types of graph elements related to the considered network instances. Calculation results are presented in Table II.

Finally, Table III contains the numbers of constrains and variables necessary to formulate the considered WDM flow design problem in relation to the selected SNDLib network instances. Number of constraints is contained in a range from 19 thousands to 26 millions. Number of variables is even larger and is contained in a range from 164 thousands to 387 millions. Such enormous numbers of constraints and variables make in practice the considered formulations numerically intractable for resolving with exact optimization methods.
TABLE III

CHARACTERISTICS OF THE FORMULATIONS

\begin{tabular}{|l|c|c|}
\hline Network instance & Constraints & Variables \\
\hline abilene & 19704 & 164388 \\
\hline atlanta & 38335 & 380855 \\
\hline brain & 26151647 & 387039416 \\
\hline cost266 & 484005 & 6226443 \\
\hline geant & 114852 & 1363998 \\
\hline germany50 & 372608 & 4766970 \\
\hline giul39 & 1263603 & 20426944 \\
\hline france & 96825 & 1106175 \\
\hline janos-us & 307484 & 4425498 \\
\hline janos-us-ca & 956135 & 14647591 \\
\hline
\end{tabular}

\section{CONCLUSION}

Paper investigates mathematical modeling of photonic networks applying wavelength division multiplexing. Based on standardization efforts, research work, and commercial offerings, a functional model of WDM network is proposed. Proposed network model involves a series of specific aspects of photonic transmission, like fiber attenuation, dispersion, and noise accumulation. Developed functional model is a basis for development of mathematical models of multicommodity flows in WDM network.

Number of integer variables used in a mathematical model in high degree determines computational complexity of optimization formulations based on this model. In case of the proposed model, this number is proportional to the squared number of WDM devices and number of WDM channels. For even small network instances (composed of several devices), this number can be at level of thousands. Thus, in the future work, in order to reduce complexity of the proposed model to numerically tractable level, authors will try to decompose it. In particular, future work will focus on adopting general decompositions methods proposed in context of large-scale linear programming, like Dantzig-Wolfe decomposition, Lagrangean relaxation, and Benders decomposition, for the case of proposed model.

\section{REFERENCES}

[1] Spectral grids for WDM applications: DWDM frequency grid. Recommendation ITU-T G.694.

[2] Interfaces for the optical transport network. Recommendation ITU-T G.709.

[3] Architecture of optical transport networks. Recommendation ITU-T G.872.

[4] Network node interface for the synchronous digital hierarchy (SDH). Recommendation ITU-T G.707.

[5] Characteristics of a single-mode optical fibre and cable. Recommendation ITU-T G.652.

[6] H. Zang, J. Jue, and B. Mukherjee, "A review of routing and wavelength assignment approaches for wavelength routed optical WDM networks,' Optical Networks Magazine, January 2000.

[7] I. Chlamtac, A. Ganz, and G. Karmi, "Lightpath communications: an approach to high bandwidth optical WAN's," IEEE Transactions on Communications, vol. 40, no. 7, pp. 1171-1182, July 1992. doi: 10.1109/26.153361. [Online]. Available: http://dx.doi.org/10.1109/26 153361 
[8] S. Evan, A. Itai, and A. Shamir, "On the complexity of timetable and multicommodity flow problems," SIAM Journal of Computing, vol. 5, pp. 691-703, 1976. doi: 10.1137/0205048. [Online]. Available: http://dx.doi.org/10.1137/0205048

[9] E. Varvarigos, K. Manousakis, and K. Christodoulopoulos, "Cross layer optimization of static lightpath demands in transparent WDM optical networks," in IEEE Information Theory Workshop on Networking and Information Theory, 2009. doi: 10.1109/ITWNIT.2009.5158553. [Online]. Available: http://dx.doi.org/10.1109/ITWNIT.2009.5158553

[10] W. Zhang, J. Tang, K. Nygard, and C. Wang, "REPARE: Regenerator placement and routing establishment in translucent networks," in IEEE Global Telecommunications Conference GLOBECOM, 2009. doi: 10.1109/GLOCOM.2009.5425649. [Online]. Available: http://dx. doi.org/10.1109/GLOCOM.2009.5425649

[11] K. Christodoulopoulos, K. Manousakis, and E. Varvarigos, "Considering physical layer impairments in offine RWA," IEEE Network, vol. 23, June 2009. doi: 10.1109/MNET.2009.4939260. [Online]. Available: http://dx.doi.org/10.1109/MNET.2009.4939260

[12] K. Manousakis, K. Christodoulopoulos, E. Kamitsas, I. Tomkos, and E. Varvarigos, "Offine impairment-aware routing and wavelength assignment algorithms in translucent WDM optica networks," Journal of Lightwave Technology, vol. 27,12, 2009. doi: 10.1109/JLT.2009.2021534. [Online]. Available: http: //dx.doi.org/10.1109/JLT.2009.2021534

[13] P. Pavon-Marino, S. Azodolmolky, R. Aparicio-Pardo, B. GarciaManrubia, Y. Pointurier, M. Angelou, J. Sole-Pareta, J. Garcia-Haro, and I. Tomkos, "Offine impairment aware RWA algorithms for crosslayer planning of optical networks," Journal of Lightwave Technology, vol. 27,12, 2009. doi: 10.1109/JLT.2009.2018291. [Online]. Available: http://dx.doi.org/10.1109/JLT.2009.2018291

[14] C. Saradhi and S. Subramaniam, "Physical layer impairment aware routing (PLIAR) in WDM optical networks: issues and challenges," Communications Surveys \& Tutorials, IEEE, vol. 11, 4, 2009. doi 10.1109/SURV.2009.090407. [Online]. Available: http://dx.doi.org/10 1109/SURV.2009.090407

[15] N. Sengezer and E. Karasan, "Static lightpath establishment in multilayer traffc engineering under physical layer impairments," IEEE/OSA Journal of Optical Communications and Networking, vol. 2, 9, 2010. doi: 10.1364/JOCN.2.000662. [Online]. Available: http://dx.doi.org/10.1364/JOCN.2.000662
[16] E. Varvarigos, K. Manousakis, and K. Christodoulopoulos, "Offlne routing and wavelength assignment in transparent WDM networks," IEEE/ACM Trans. on Networks, vol. 18,5, 2010. doi: 10.1109/TNET.2010.2044585. [Online]. Available: http://dx.doi.org/10.1109/TNET.2010.2044585

[17] Y. Zhai, A. Askarian, S. Subramaniam, Y. Pointurier, and M. Brandtpearce, "Cross-layer approach to survivable DWDM network design," IEEE/OSA Journal of Optical Communications and Networking, vol. 2,6, 2010. doi: 10.1364/JOCN.2.000319. [Online]. Available: http://dx.doi.org/10.1364/JOCN.2.000319

[18] R. Aparicio-Pardo, M. Klinkowski, B. Garcia-Manrubia, P. PavonMarino, and D. Careglio, "Offine impairment-aware rwa and regenerator placement in translucent optical networks," Journal of Lightwave Technology, vol. 29,3, 2011. doi: 10.1109/JLT.2010.2098393. [Online]. Available: http://dx.doi.org/10.1109/JLT.2010.2098393

[19] N. Sengezer and E. Karasan, "Multi-layer virtual topology design in optical networks under physical layer impairments and multi-hour traffc demand," EEE/OSA Journal Journal of Optical Communications and Networking, vol. 4,2, 2012. doi: 10.1364/JOCN.4.000078. [Online]. Available: http://dx.doi.org/10.1364/JOCN.4.000078

[20] J. Sole, S. Subramaniam, D. Careglio, and S. Spadaro, "Cross-layer approaches for planning and operating impairmentaware optical networks," in Proc. the IEEE 100, 2012 doi: 10.1109/JPROC.2012.2185669. [Online]. Available: http: //dx.doi.org/10.1109/JPROC.2012.2185669

[21] M. Minoux, Mathematical Programming: Theory and Algorithms. John Wiley \& Sons, 1986.

[22] R. K. Ahuja, T. L. Magnanti, and J. B. Orlin, Network Flows: Theory Algorithms, and Applications. Prentice Hall, 1993.

[23] J. Strand, A. Chiu, and R. Tkach, "Issues for routing in the optical layer," IEEE Communications Magazine, 2001. doi: 10.1109/35.900635. [Online]. Available: http://dx.doi.org/10.1109/35.900635

[24] M. Dzida, n. T. Sliwi M. Zagozdzon, W. Ogryczak, and M. Pioro, "Path generation for a class survivable network design problems," in NGI 2008 Conference on Next Generation Internet Networks, Cracow, Poland, 2008. doi: 10.1109/NGI.2008.11. [Online]. Available: http://dx.doi.org/10.1109/NGI.2008.11

[25] "SNDlib 1.0 - Survivable network design data library," 2005. [Online]. Available: http://sndlib.zib.de 